\title{
Erratum
}

\section{Inhibitory Effect of Chlorophyllin on the Propionibacterium acnes-Induced Chemokine Expression}

\author{
Mi-Sun Kang ${ }^{1}$, Jin-Hee Kim ${ }^{1}$, Boo-Ahn Shin ${ }^{1}$, Hyun-Chul Lee ${ }^{1}$, Youn-Shin Kim ${ }^{2}$, \\ Hae-Soon $\mathrm{Lim}^{3}$, and Jong-Suk $\mathrm{Oh}^{1 *}$ \\ ${ }^{1}$ Department of Microbiology, School of Medicine, Chonnam National University, Gwangju 501-746, Republic of Korea \\ ${ }^{2}$ Department of Forensic Medicine, School of Medicine, Chosun University, Gwangju 501-759, Republic of Korea \\ ${ }^{3}$ Department of Dental Education, School of Dentistry, Chonnam National University, Gwangju 500-757, Republic of Korea
}

In the article by Kang et al. published in Journal of Microbiology 2013; 51, 844-849. Figure 4 on page 847 should be changed as below.

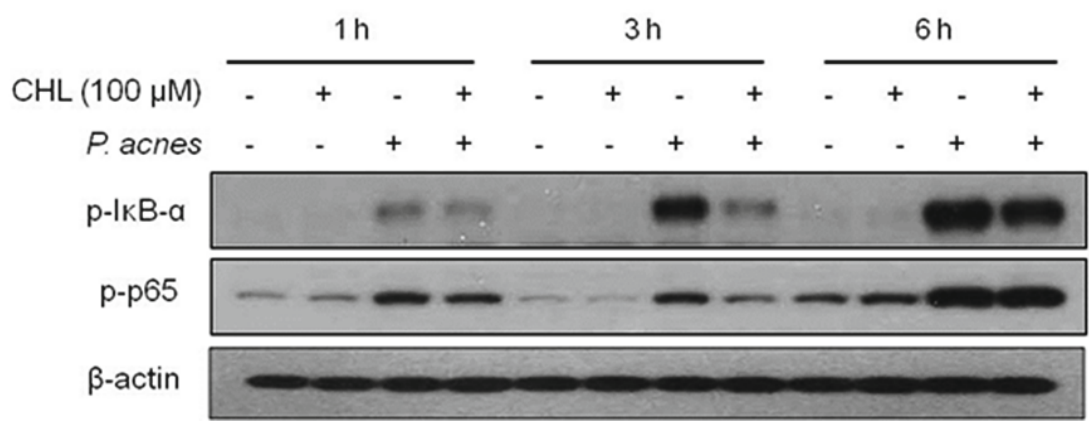

